International Journal of Pure and Applied Mathematics

Volume $90 \quad$ No. 2 2014, 189-193

ISSN: 1311-8080 (printed version); ISSN: 1314-3395 (on-line version)

url: http://www.ijpam.eu

doi: http://dx.doi.org/10.12732/ijpam.v90i2.8

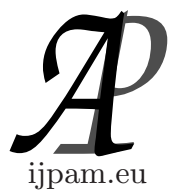

\title{
ON A NOTE OF CYCLE-AXIOM OF HYPERGRAPHS
}

\author{
Wei Feng $^{1}$, Lingqi Zhao ${ }^{2}$, Chunlei $\mathrm{Xu}^{3}$, Jirimutu ${ }^{4}$ \\ ${ }^{1,4}$ College of Mathematics \\ Inner Mongolian University for Nationalities \\ Tongliao, 028043, P.R. CHINA \\ ${ }^{2,3}$ College of Computer Science and Technology \\ Inner Mongolian University for Nationalities \\ Tongliao, 028043, P.R. CHINA
}

\begin{abstract}
The cycle-structure of hypergraph plays an important role in the hypergraph theory and the database theory. It has been well studied during last several years. Especially in recent years, many researchers continue to study. Wang introduced the cycle-axiom of hypergraph. In this paper, we make a note of cycle-axiom of hypergraph to explain that the cycle-axiom of hypergraph is reasonable, and displays an important role in the hypergraph theory.
\end{abstract}

AMS Subject Classification: 05C65

Key Words: hypergraph, cycle, cycle-axiom

\section{Introduction}

Hypergraphs are subsets systems of finite sets and may be regarded as the most general structures in discrete mathematics. Combinatorists of 1960s developed a systematic combinatorial approach of finite sets and suggested the term "Hypergraph". As extension of graphs, there are many results on trees, cycles, covering and coloring of hypergraph, see $[4,5]$. With the emergence of informa-

Received: September 30, 2013

(C) 2014 Academic Publications, Ltd.

${ }^{\S}$ Correspondence author url: www.acadpubl.eu 
tional science and life science, size of systems we deal with is becoming bigger and bigger. In early 1980s, informational scientists introduced decompositionjoin approach into the design and study of databases with large size. As Lee [6] pointed out, "The core issue in the theory of database dependence is information preserving decomposition of a relation into several subrelations in such a way that the original relation can be regenerated by join operation." A decomposition of a relation induces a database scheme, that is a hypergraph on the attributer set. As Beeri et al [3] pointed out, "Berge-acyclicity is too restrictive an assumption to make about database schemes."Beeri et al [7] introduced a special class of database schemes, called acyclic. Fagin et al [8] have shown that this class enjoys a certain desirable property. Beeri et al [3] have identified a number of other desirable properties, which have been studied by other researchers in quite different contexts, and show that each of those properties is equivalent to acyclicity. Thus the class of acyclic database schemes is natural, important class, since it can be characterized in a number of ways, each corresponding to a desirable property of database schemes or to a natural graph-theoretic property. Wang [9] first introduced the acyclic-axiom of hypergraph, and listed several theoretical results for acyclic hypergraph to demonstrate the usefulness of acyclic-axiom, then introduced the cycle-axiom in order to improve the definition of cycles in hypergraph, finally showed that the cycle-axiom includes the acyclic-axiom. In this paper, we make a note of cycle-axiom of hypergraph to explain that the cycle-axiom of hypergraph is reasonable, and displays an important role in the hypergraph theory.

\section{The Further Comprehension of the Cycle-Axiom of Hypergraph}

Definition. (see [9]) Let $V$ be a finite set, $\varepsilon \subseteq 2^{V}, \mathrm{H}=(V, \varepsilon)$, simply $\varepsilon$ is called a hypergraph on $V$, if $\forall e \in \varepsilon, e \neq \phi$, and $\bigcup_{e \in \varepsilon} e=V$, the elements in $V$ and $\varepsilon$ are called vertices and edges, respectively.

Cycle-Axiom. (see [9]) Let $\varepsilon$ be a hypergraph with at least three edges, $C=\left(e_{0}, e_{1}, \cdots, e_{k-1}\right)$ be an edge-sequence of $\varepsilon, S_{i}=e_{i} \bigcap e_{i+1}, i \in \mathbf{Z}_{k}$. We call $C$ a cycle of $\varepsilon$ if for any $i \in \mathbf{Z}_{k}$ and $\forall e \in \varepsilon,\left(S_{i-1} \bigcup S_{i} \bigcup S_{i+1}\right) \backslash e \neq \phi$.

If changing the condition $i \in \mathbf{Z}_{k}$ in the above cycle-axiom, we can obtain the following proposition.

Proposition. Let $\varepsilon$ be a hypergraph. A cycle of $\varepsilon$ is a sequence of edges $\left(e_{i_{1}}, e_{i_{2}}, \cdots, e_{i_{\mathrm{k}}}\right)$ satisfying the following conditions:

1) $e_{i_{1}}=e_{i_{\mathrm{k}}}$ 
2) $\forall 2 \leq j \leq k-2$ and $\forall e \in \varepsilon,\left(S_{j-1} \bigcup S_{j} \bigcup S_{j+1}\right) \backslash e \neq \phi$, with $S_{j}=$ $e_{i \mathrm{j}} \cap e_{i_{\mathrm{j}+1}}, \forall 1 \leq j \leq k-1$.

It seems that the cycles satisfying the above proposition is equivalent to the cycle satisfying cycle-axiom, but they aren't equivalent actually. We give an example to explain the non-equivalence. Given $e_{i_{1}}=\{1,2,3,4\}, e_{i_{2}}=$ $\{2,3,4,5\}, e_{i_{3}}=\{3,4,5,6\}, e_{i_{4}}=\{2,3,5,6\}$, and let $E_{1}=\left(e_{i_{1}}, e_{i_{2}}, e_{i_{3}}, e_{i_{4}}, e_{i_{5}}\right)$ (where $\left.e_{i_{5}}=e_{i_{1}}\right), E_{2}=\left(e_{i_{3}}, e_{i_{4}}, e_{i_{1}}, e_{i_{2}}, e_{i_{3}}\right)$. According to the above proposition, it is easy to verify that the sequence of edges $E_{1}$ is a cycle, $E_{2}$ is not a cycle. We notice that $E_{1}$ and $E_{2}$ contain the same elements in terms of set. In other words, a sequence of edges is whether or not a cycle which is related to its starting point and end point, which is not logical. It doesn't appear this case in cycle-axiom of hypergraph. The reason is that the subscript $i$ in the cycle-axiom and $j$ in the proposition take different values, in other words, $j$ lacks modular arithmetic.

A cycle defined by the cycle-axiom, which is related to the global structure of the hypergraph. For example, given $\varepsilon_{1}=\{a b c, c d e, e f a\}, \varepsilon_{2}=\{a b c$, cde, ef $a$, $a c e\}$ are two hypergraphs, $E=(a b c, c d e$, efa $)$ is a sequence of edges in $\varepsilon_{1}$ and $\varepsilon_{2}$. It is easy to verify that $E$ is a cycle satisfying cycle-axiom in $\varepsilon_{1}$, but it is not a cycle in $\varepsilon_{2}\left(=\varepsilon_{1} \bigcup\{a c e\}\right)$. So a sequence of edges is whether or not a cycle, which is related to the global structure of hypergraph. So we say that the cycle-axiom characters the global structure of hypergraph, see [10].

The cycle-axiom of hypergraph, which characters the local structure of hypergraph too, see [10]. It implies three case:

(a) $\forall i \in \mathbf{Z}_{k}, S_{i} \neq \phi$.

(b) $\forall i \in \mathbf{Z}_{k}, S_{i} \backslash S_{i+1} \neq \phi$, and $S_{i+1} \backslash S_{i} \neq \phi$.

(c) $\forall i \in \mathbf{Z}_{k}, S_{i} \backslash S_{i+2} \neq \phi$, and $S_{i+2} \backslash S_{i} \neq \phi$.

A graph, is regarded as a hypergraph whose any edge contains two vertices. The cycle-axiom defines also cycles of graphs, but a cycle of a graph degenerates to a local structure without any relation with other parts of the graph, see [10]. There are two aspects explain this:

(1) Since every edge contains exactly two vertices in a graph, so the cycleaxiom is still true, i.e. the cycle-axiom can determines that any sequence of edges in graph is whether or not a cycle.

(2) Given $G$ is a graph, $C$ is a cycle in $G$. If adding an edge $e$ to $G, C$ is still a cycle in the new graph $G \bigcup\{e\}$, while $C$ degenerates to a local structure without any relation with other parts of the graph. The cycle-axiom shows that graphs are degenerated case of hypergraph, see [10]. 


\section{Conclusion}

As Wang in [10] pointed out: In algebra, there are similar model, for example, normal subgroups of groups, ideals of rings, which play an important and fundamental role in corresponding subjects. The cycle defined by cycle-axiom is not only a local structure, but also has relations with the global structure of the hypergraph. The condition of cycle-axiom, not only characterizes the local structure of a cycle in hypergraph, but also displays the inter-relations between it and other parts of the global hypergraph. It may capture the realworld situations in information systems. This is a new idea and model in discrete mathematics. The cycle-axiom also defines the cycles of graph. So the cycle-axiom of hypergraph is reasonable, and displays an important role in the hypergraph theory. It will develop discrete mathematics to a new stage.

\section{Acknowledgments}

The authors are grateful to Jian-fang Wang, Philippe Jégou, and Samba Ndojh Ndiaye for helpful comments.

The research is supported by the National Natural Science Foundation (1116 1032 ) and (61262018). The research is supported by Inner Mongolia Department of Education Projects (NJZY11209) and (NJZY13175). The research is supported by Inner Mongolian University for Nationalities Projects (NMD1104) and (NMD1123).

This research is supported by Institute for Discrete mathematics of Inner Mongolia University for Nationalities.

\section{References}

[1] Jian-Fang Wang, The theoretical base of hypergraphs, in Chinese, Beijing, Higher Education Press (2006).

[2] Philippe Jégou, Samba Ndojh Ndiaye, On the notion of cycles in hypergraphs, Discrete Mathematics, 309, No-s: 23-24 (2009), 6535-6543.

[3] Ronald Fagin, Degrees of acyclicity for hypergraphs and relational database schemes, Journal of the Assoeiauon for Computing Machinery, 30, No. 3 (1983), 514-550.

[4] C. Berge, Hypergraphs, North-Holland, Amsterdam, 1989. 
[5] P. Duchet, Hypergraphs, Handbook of Combinatorics, The MIT Press (1995), 381-423.

[6] T.T. Lee, An Information - Theoretic Analysis of Relational Databases - Part I. Part II, IEE Transactions on Software Engineering, 13 (1987), 1049-1072.

[7] C. Beeri, R. Fagin, D. Maier, A.O. Mendelzon, J.D. Ullman, M. Yannakis, Properties of acyclic database schemes, In: Proc. 13-th Ann. ACM Symp. on Theory of Computing, Milwaukee, Wise, May 11-13, 1981, ACM, New York (1981), 355-362.

[8] R. Fagin, A.O. Mendelzon, J.D. Ullman, A simplified universal relation assumption and its properties, ACM Trans. Database Syst., 7, No. 3 (1982), 343-360.

[9] Jian-Fang Wang, On axioms contituting the foundation of hypergraphs theory, Acta Mathematicae Applicatae Sinica, English Series, 21, No. 3 (2005), 495-498.

[10] Jian-Fang Wang, The Information Hypergraph Theory, Beijing, Science Press (2008). 
